International Journal of Pure and Applied Mathematics

Volume 88 No. 2 2013, 297-304

ISSN: 1311-8080 (printed version); ISSN: 1314-3395 (on-line version)

url: http://www.ijpam.eu

doi: http://dx.doi.org/10.12732/ijpam.v88i2.11

ijpam.eu

\title{
COMMON FIXED POINT THEOREMS OF WEAK RECIPROCAL CONTINUITY IN METRIC SPACES
}

\author{
Saurabh Manro ${ }^{1}$, Sanjay Kumar², \\ Satwinder Singh Bhatia ${ }^{3}$, Shin Min Kang $4 \S$ \\ ${ }^{1,3}$ School of Mathematics and Computer Applications \\ Thapar University \\ Patiala, 147004, Punjab, INDIA \\ ${ }^{2}$ Department of Mathematics \\ Deenbandhu Chhotu Ram University of Science and Technology \\ Murthal, 131039, Sonepat, INDIA \\ ${ }^{4}$ Department of Mathematics and RINS \\ Gyeongsang National University \\ Jinju, 660-701, KOREA
}

\begin{abstract}
In this paper, we prove common fixed point theorems for contraction mappings, which generalizes the result of Pant et al [Weak reciprocal continuity and fixed point theorems, Ann. Univ. Ferrara, 57 (2011), 181-190] using the concept of weak reciprocal continuity in metric spaces.
\end{abstract}

AMS Subject Classification: 47H10, 54H25

Key Words: weak reciprocal continuity, compatible, $R$-weakly commuting, $R$-weakly commuting of type $\left(A_{f}\right)$, of type $\left(A_{g}\right)$ and of type $(P)$

\section{Introduction and Preliminaries}

In 1922, Banach proved a common fixed point theorem, which ensures under

Received: August 7, 2013

(C) 2013 Academic Publications, Ltd.

${ }^{\S}$ Correspondence author url: www.acadpubl.eu 
appropriate conditions, the existence and uniqueness of a fixed point. This result of Banach is known as Banach's fixed point theorem or Banach Contraction Principle. Many authors have extended, generalized and improved the Banach's fixed point theorem in different ways.

Jungck [1] proved a common fixed point theorem for commuting mappings, which generalizes the Banachs fixed point theorem. This Many aurthors has generalized this theorem, but suffers from one drawback that the continuity of mapping throughout the space.

Jungck [2] defined the concept of compatible mappings.

Definition 1.1. Let $f$ and $g$ be two self-mappings of a metric space $(X, d)$. Then $f$ and $g$ are is said to be compatible if $\lim _{n \rightarrow \infty} d\left(f g x_{n}, g f x_{n}=0\right.$ whenever $\left\{x_{n}\right\}$ is a sequence in $X$ such that $\lim _{n \rightarrow \infty} f x_{n}=g x_{n}=z$ for some $z \in X$.

An immediate consequence [2, Proposition 2.2] is that if $f$ and $g$ are compatible and $f z=g z$ ( $z$ is called a coincidence point of $f$ and $g$ ), then $f g z=g f z$.

In 1994, Pant [4] introduced the notion of $R$-weak commutativity in a metric space.

Definition 1.2. Let $f$ and $g$ be two self-mappings of a metric space $(X, d)$. Then $f$ and $g$ are called $R$-weakly commuting on $X$ if there exists $R>0$ such that $d(f g x, g f x) \leq R d(f x, g x)$ for all $x \in X$.

In 1997, Pathak et al. [7] generalized the notion of $R$-weakly commuting mappings to $R$-weakly commuting mappings of type $\left(A_{f}\right)$ and of type $\left(A_{g}\right)$.

Definition 1.3. Let $f$ and $g$ be two self-mappings of a metric space $(X, d)$. Then $f$ and $g$ are called $R$-weakly commuting of type $\left(A_{g}\right)$ if there exists $R>0$ such that $d(f f x, g f x) \leq R d(f x, g x)$ for all $x \in X$.

Similarly, the two self-mappings $f$ and $g$ are called $R$-weakly commuting of type $\left(A_{f}\right)$ if there exists $R>0$ such that $d(f g x, g g x) \leq R d(f x, g x)$ for all $x \in X$.

It is obvious that $R$-weakly commuting mappings commute at their coincidence points and $R$-weak commutativity is equivalent to commutativity at coincidence points. It may be noted that both compatible and non-compatible mappings can be $R$-weakly commuting of type $\left(A_{g}\right)$ or $\left(A_{f}\right)$, but the convse is not true.

Definition 1.4. ([3]) Let $f$ and $g$ be two self-mappings of a metric space $(X, d)$. Then $f$ and $g$ are called $R$-weakly commuting of type $(P)$ if there exists $R>0$ such that $d(f f x, g g x) \leq R d(f x, g x)$ for all $x \in X$.

In 1998, Pant [5] introduced a new continuity condition, known as reciprocal 
continuity and obtained a common fixed point theorem by using the compatibility in a metric space and also showed that in the setting of common fixed point theorems for compatible mappings satisfying contraction conditions, the notion of reciprocal continuity is weaker than the continuity of one of the mappings. Also, the notion of pointwise $R$-weakly commuting mappings made the scope of the study of common fixed point theorems from the class of compatible to the wider class of pointwise $R$-weakly commuting mappings. Subsequently, several common fixed point theorems have been proved by combining the ideas of $R$ weakly commuting mappings and reciprocal continuity of mappings in different settings.

Definition 1.5. Let $f$ and $g$ be two self-mappings of a metric space $(X, d)$. Then $f$ and $g$ are called reciprocally continuous if $\lim _{n \rightarrow \infty} f g x_{n}=f z$ and $\lim _{n \rightarrow \infty} g f x_{n}=g z$, whenever $\left\{x_{n}\right\}$ is a sequence such that $\lim _{n \rightarrow \infty} f x_{n}=\lim _{n \rightarrow \infty} g x_{n}=$ $z$ for some $z \in X$.

If $f$ and $g$ are both continuous, then they are obvious reciprocally continuous but the converse is not true ([5]).

Recently, Pant et al. [6] generalized the notion of reciprocal continuity to weak reciprocal continuity as follows:

Definition 1.6. Let $f$ and $g$ be two self-mappings of a metric space $(X, d)$. Then $f$ and $g$ are called weakly reciprocally continuous if $\lim _{n \rightarrow \infty} f g x_{n}=f z$ or $\lim _{n \rightarrow \infty} g f x_{n}=g z$, whenever $\left\{x_{n}\right\}$ is a sequence such that $\lim _{n \rightarrow \infty} f x_{n}=\lim _{n \rightarrow \infty} g x_{n}=$ $z$ for some $z \in X$.

If $f$ and $g$ are reciprocally continuous, then they are obviously weakly reciprocally continuous but the converse is not true ([6]).

As an application of weak reciprocal continuity, we prove common fixed point theorems under contractive conditions that extend the scope of the study of common fixed point theorems from the class of compatible continuous mappings to a wider class of mappings which also includes non-compatible and discontinuous mappings.

In this paper, we prove common fixed point theorems for contraction mappings, which generalizes the result of Pant et al. [6] using the concept of weak reciprocal continuity in metric spaces.

\section{Main Results}

Now, we are ready to give our main theorem. 
Theorem 2.1. Let $f$ and $g$ be weakly reciprocally continuous self-mappings of a complete metric space $(X, d)$ satisfying

(C1) $f(X) \subset g(X)$,

$(C 2)$ there exists a number $h \in(0,1)$ such that

$$
\begin{gathered}
d(f x, f y) \leq h \max \{d(g x, g y), d(f x, g x), d(f y, g y)), \\
[d(f x, g y)+d(f y, g x)] / 2\}
\end{gathered}
$$

for any $x, y \in X$.

If $f$ and $g$ are either compatible or $R$-weakly commuting of type $\left(A_{g}\right)$ or $R$-weakly commuting of type $\left(A_{f}\right)$ or $R$-weakly commuting of type $(P)$, then $f$ and $g$ have a unique common fixed point.

Proof. Let $x_{0}$ be any point in $X$. Then as $f(X) \subset g(X)$, we can define a sequence $\left\{y_{n}\right\}$ in $X$ as

$$
y_{n}=f x_{n}=g x_{n+1} .
$$

Now, we show that $\left\{y_{n}\right\}$ is a Cauchy sequence in $X$. For proving this, by $(C 2)$, we have

$$
\begin{gathered}
d\left(y_{n}, y_{n+1}\right)=d\left(f x_{n}, f x_{n+1}\right) \\
\leq h \max \left\{d\left(g x_{n}, g x_{n+1}\right), d\left(f x_{n}, g x_{n}\right), d\left(f x_{n+1}, g_{n+1}\right),\right. \\
\left.\left[d\left(f x_{n}, g x_{n+1}\right)+d\left(f x_{n+1}, g x_{n}\right)\right] / 2\right\} \\
\leq h \max \left\{d\left(y_{n-1}, y_{n}\right), d\left(y_{n}, y_{n-1}\right), d\left(y_{n+1}, y_{n}\right),\right. \\
\left.\left[d\left(y_{n+1}, y_{n}\right)+d\left(y_{n}, y_{n-1}\right)\right] / 2\right\} .
\end{gathered}
$$

If $d\left(y_{n}, y_{n+1}\right)>d\left(y_{n-1}, y_{n}\right)$, then we have

$$
d\left(y_{n}, y_{n+1}\right) \leq h d\left(y_{n}, y_{n+1}\right),
$$

which is a contraction. Thus we have

$$
d\left(y_{n}, y_{n+1}\right) \leq h d\left(y_{n-1}, y_{n}\right)
$$

Further we can obtain

$$
\begin{aligned}
d\left(y_{n}, y_{n+1}\right) & \leq h d\left(y_{n-1}, y_{n}\right) \\
& \leq h^{2} d\left(y_{n-2}, y_{n-1}\right) \\
& \leq \cdots \\
& \leq h^{n} d\left(y_{0}, y_{1}\right) .
\end{aligned}
$$


Therefore, for all $n, m \geq \mathbb{N}$ (: set of natural numbers), $n<m$, we have

$$
\begin{aligned}
d\left(y_{n}, y_{m}\right) \leq & d\left(y_{n}, y_{n+1}\right)+d\left(y_{n+1}, y_{n+2}\right)+d\left(y_{n+2}, y_{n+3}\right) \\
& +\cdots+d\left(y_{m-1}, y_{m}\right) \\
\leq & \left(h^{n}+h^{n+1}+h^{n+2}+\cdots+h^{m-1}\right) d\left(y_{0}, y_{1}\right) \\
\leq & \left(h^{n}+h^{n+1}+h^{n+2}+\cdots\right) d\left(y_{0}, y_{1}\right) \\
= & \frac{h^{n}}{1-h} d\left(y_{0}, y_{1}\right) \rightarrow 0
\end{aligned}
$$

as $n \rightarrow \infty$. Thus $\left\{y_{n}\right\}$ is a Cauchy sequence in $X$. As $X$ is complete, there exists a point $z$ in $X$ such that $\lim _{n \rightarrow \infty} y_{n}=z$. Therefore, by (2.1), we have

$$
\lim _{n \rightarrow \infty} y_{n}=\lim _{n \rightarrow \infty} f x_{n}=\lim _{n \rightarrow \infty} g x_{n+1}=z .
$$

Suppose that $f$ and $g$ are compatible mappings. Now, by weak reciprocal continuity of $f$ and $g$ implies that $\lim _{n \rightarrow \infty} f g x_{n}=f z$ or $\lim _{n \rightarrow \infty} g f x_{n}=g z$.

Let $\lim _{n \rightarrow \infty} g f x_{n}=g z$. Then compatibility of $f$ and $g$ gives $\lim _{n \rightarrow \infty} d\left(f g x_{n}, g f x_{n}\right)$ $=0$, that is, $\lim _{n \rightarrow \infty} d\left(f g x_{n}, g z\right)=0$. Hence $\lim _{n \rightarrow \infty} f g x_{n}=g z$. By (2.1), we get $\lim _{n \rightarrow \infty} f g x_{n+1}=\lim _{n \rightarrow \infty} f f x_{n}=g z$.

Therefore, by $(C 2)$, we get

$$
\begin{gathered}
d\left(f z, f f x_{n}\right) \leq h \max \left\{d\left(g z, g f x_{n}\right), d(f z, g z), d\left(f f x_{n}, g f x_{n}\right),\right. \\
\left.\left[d\left(f z, g f x_{n}\right)+d\left(f f x_{n}, g z\right)\right] / 2\right\} .
\end{gathered}
$$

As $n \rightarrow \infty$, since $h<1$

$$
\begin{aligned}
d(f z, g z) & \leq h \max \{0, d(f z, g z), 0, d(f z, g z) / 2\} \\
& =h d(f z, g z),
\end{aligned}
$$

which is a contraction, so that, we get $f z=g z$. Again compatibility of $f$ and $g$ implies commutativity at a coincidence point. Hence $g f z=f g z=f f z=g g z$. Using (C2) we obtain

$$
\begin{aligned}
d(f z, f f z) \leq h \max \{d(g z, g f z), d(f z, g z), d(f f z, g f z), & {[d(f z, g f z)+d(f f z, g z)] / 2\} } \\
= & h \max \{d(f z, f f z), 0,0,[d(f z, f f z)+d(f f z, f z)] / 2\},
\end{aligned}
$$

which implies that $f z=f f z$. Hence $f z=f f z=g f z$ and $f z$ is a common fixed point of $f$ and $g$. 
Next suppose that $\lim _{n \rightarrow \infty} f g x_{n}=f z$. Then by $f(X) \subset g(X)$, there exists $u \in X$ such that $f z=g u$ and $\lim _{n \rightarrow \infty} f g x_{n}=g u$. Compatibility of $f$ and $g$ implies $\lim _{n \rightarrow \infty} g f x_{n}=g u$. By virtue of (2.1), this gives $\lim _{n \rightarrow \infty} f g x_{n+1}=\lim _{n \rightarrow \infty} f f x_{n}=g u$. Using $(C 2)$, we get

$$
\begin{gathered}
d\left(f u, f f x_{n}\right) \leq h \max \left\{d\left(g u, g f x_{n}\right), d(f u, g u), d\left(f f x_{n}, g f x_{n}\right),\right. \\
\left.\left[d\left(f u, g f x_{n}\right)+d\left(f f x_{n}, g u\right)\right] / 2\right\} .
\end{gathered}
$$

As $n \rightarrow \infty$,

$$
d(f u, g u) \leq h \max \{0, d(f u, g u), 0, d(f u, g u) / 2\}
$$

so that get $f u=g u$. Compatibility of $f$ and $g$ yields $f g u=g g u=f f u=g f u$. Finally, using $(C 2)$, we obtain

$$
\begin{aligned}
& d(f u, f f u) \leq h \max \{d(g u, g f u), d(f u, g u), d(f f u, g f u), {[d(f u, g f u)+d(f f u, g u)] / 2\} } \\
&=h \max \{d(f u, f f u), 0,0,[d(f u, f f u)+d(f f u, f u)] / 2\},
\end{aligned}
$$

that is, $f u=f f u$. Hence $f u=f f u=g f u$ and $f u$ is a common fixed point of $f$ and $g$.

Now, suppose that $f$ and $g$ are $R$-weakly commuting of type $\left(A_{g}\right)$. Now, weak reciprocal continuity of $f$ and $g$ implies that $\lim _{n \rightarrow \infty} f g x_{n}=f z$ or $\lim _{n \rightarrow \infty} g f x_{n}=$ $g z$.

Assume that $\lim _{n \rightarrow \infty} g f x_{n}=g z$. Then $R$-weak commutativity of type $\left(A_{g}\right)$ of $f$ and $g$ yields $d\left(f f x_{n}, g f x_{n}\right) \leq R d\left(f x_{n}, g x_{n}\right)$, that is, $\lim _{n \rightarrow \infty} d\left(f f x_{n}, g z\right)=0$. This gives $\lim _{n \rightarrow \infty} f f x_{n}=g z$. Also, using $(C 2)$, we get

$$
\begin{gathered}
d\left(f z, f f x_{n}\right) \leq h \max \left\{d\left(g z, g f x_{n}\right), d(f z, g z), d\left(f f x_{n}, g f x_{n}\right),\right. \\
\left.\left[d\left(f z, g f x_{n}\right)+d\left(f f x_{n}, g z\right)\right] / 2\right\} .
\end{gathered}
$$

As $n \rightarrow \infty$,

$$
d(f z, g z) \leq h \max \{0, d(f z, g z), 0, d(f z, g z) / 2\}
$$

so that we get $f z=g z$. Again, by using $R$-weak commutativity of type $\left(A_{g}\right)$,

$$
d(f f z, g f z) \leq h d(f z, g z)=0 .
$$

This yields $f f z=g f z$. Therefore $f f z=f g z=g f z=g g z$. Using $(C 2)$, we get

$$
\begin{aligned}
& d(f z, f f z) \leq h \max \{d(g z, g f z), d(f z, g z), d(f f z, g f z), {[d(f z, g f z)+d(f f z, g z)] / 2\} } \\
& \leq h \max \{d(f z, f f z), 0,0,[d(f z, f f z)+d(f f z, f z)] / 2\},
\end{aligned}
$$


that is, we have $f z=f f z$. Hence $f z=f f z=g f z$ and $f z$ is a common fixed point of $f$ and $g$.

Similarly, if $\lim _{n \rightarrow \infty} f g x_{n}=f z$, then we can easily prove.

Suppose that $f$ and $g$ are $R$-weakly commuting of type $\left(A_{f}\right)$. Again, as done above, we can easily prove that $f z$ is a common fixed point of $f$ and $g$.

Finally, suppose $f$ and $g$ are $R$-weakly commuting of type $(P)$. Weak reciprocal continuity of $f$ and $g$ implies that $\lim _{n \rightarrow \infty} f g x_{n}=f z$ or $\lim _{n \rightarrow \infty} g f x_{n}=g z$.

Let us assume that $\lim _{n \rightarrow \infty} g f x_{n}=g z$. Then $R$-weak commutativity of type $(P)$ of $f$ and $g$ yields $d\left(f f x_{n}, g g x_{n}\right) \leq R d\left(f x_{n}, g x_{n}\right)$, that is, $\lim _{n \rightarrow \infty} d\left(f f x_{n}, g g x_{n}\right)$ $=0$. Using (2.1), we have $g f x_{n-1}=g g x_{n} \rightarrow g z$ and $f f x_{n} \rightarrow g z$ as $n \rightarrow \infty$. Also, using $(C 2)$ we get

$$
\begin{gathered}
d\left(f z, f f x_{n}\right) \leq h \max \left\{d\left(g z, g f x_{n}\right), d(f z, g z), d\left(f f x_{n}, g f x_{n}\right),\right. \\
\left.\left[d\left(f z, g f x_{n}\right)+d\left(f f x_{n}, g z\right)\right] / 2\right\} .
\end{gathered}
$$

As $n \rightarrow \infty$,

$$
d(f z, g z) \leq h \max \{0, d(f z, g z), 0, d(f z, g z) / 2\}
$$

so that $f z=g z$. Again, by using $R$-weak commutativity of type $(P), d(f f z, g g z)$ $\leq R d(f z, g z)=0$. This yields $f f z=g g z$. Therefore $f f z=f g z=g f z=g g z$. Using $(C 2)$, we get

$$
\begin{aligned}
& d(f z, f f z) \leq h \max \{d(g z, g f z), d(f z, g z), d(f f z, g f z) \\
& {[d(f z, g f z)+d(f f z, g z)] / 2\} } \\
& \leq h \max \{d(f z, f f z), 0,0,[d(f z, f f z)+d(f f z, f z)] / 2\}
\end{aligned}
$$

so that $f z=f f z$. Hence $f z=f f z=g f z$ and $f z$ is a common fixed point of $f$ and $g$.

Similarly, if $\lim _{n \rightarrow \infty} f g x_{n}=f z$, then we can easily prove.

Uniqueness of the common fixed point theorem follows easily in each of the four cases by using $(C 2)$.

Remark 2.2. Theorem 2.1 generalizes the result of Pant et al. [6]. Indeed, the condition $(\mathrm{C} 2)$ is more general than the contractive condition of Pant et al. [6].

We now give an example to illustrate Theorem 2.1. 
Example 2.3. ([6]) Let $(X, d)$ be a metric space, where $X=[2,20]$ and $d(x, y)=|x-y|$ for all $x, y \in X$. Define $f, g: X \rightarrow X$ by

$$
\left\{\begin{array} { l l } 
{ f x = 2 } & { \text { if } x = 2 \text { or } x > 5 , } \\
{ f x = 6 } & { \text { if } 2 < x \leq 5 , }
\end{array} \quad \left\{\begin{array}{ll}
g x=2 & \text { if } x=2, \\
g x=11 & \text { if } 2<x \leq 5 \\
g x=(x+1) / 3 & \text { if } x>5
\end{array}\right.\right.
$$

Then it can be verified that $f$ and $g$ satisfy the contractive condition $(C 2)$ with $4 / 9 \leq h<1$.

Let $\left\{x_{n}\right\}$ be a sequence in $X$ such that either $x_{n}=2$ or $x_{n}=5+1 / n$ for each $n$. Then $f$ and $g$ satisfy all the conditions of Theorem 2.1 and have a unique common fixed point at $x=2$.

\section{References}

[1] G. Jungck, Commuting mappings and fixed points, Amer. Math. Monthly, 83, No. 4 (1976), 261-263, doi: 10.2307/2318216.

[2] G. Jungck, Compatible mappings and common fixed points, Int. J. Math. Math. Sci., 9, No. 4 (1986), 771-779, doi: 10.1155/S0161171286000935.

[3] S. Kumar, S.K. Garg, Expansion mappings theorems in metric spaces, Int. J. Contemp. Math. Sci., 4, No. 36 (2009), 1749-1758.

[4] R.P. Pant, Common fixed points of non-commuting mappings, J. Math. Anal. Appl., 188, No. 2 (1994), 436-440, doi: 10.1006/jmaa.1994.1437

[5] R.P. Pant, Common fixed points of four mappings, Bull. Cal. Math. Soc., 90, No. 4 (1998), 281-286.

[6] R.P. Pant, R.K. Bisht, D. Arora, Weak reciprocal continuity and fixed point theorems, Ann. Univ. Ferrara, 57, No. 1 (2011), 181-190, doi: 10.1007/s11565-011-0119-3.

[7] H.K. Pathak, Y.J. Cho, S.M. Kang, Remarks of $R$-weakly commuting mappings and common fixed point theorems, Bull. Korean Math. Soc., 34, No. 2 (1997), 247-257. 